Tenner, Uwe; Erné, Sergio N.; Haueisen, Jens; Nowak, Hannes; Brauer, Hartmut:

Physical Phantoms for Biomagnetic and Bioelectric Measurements

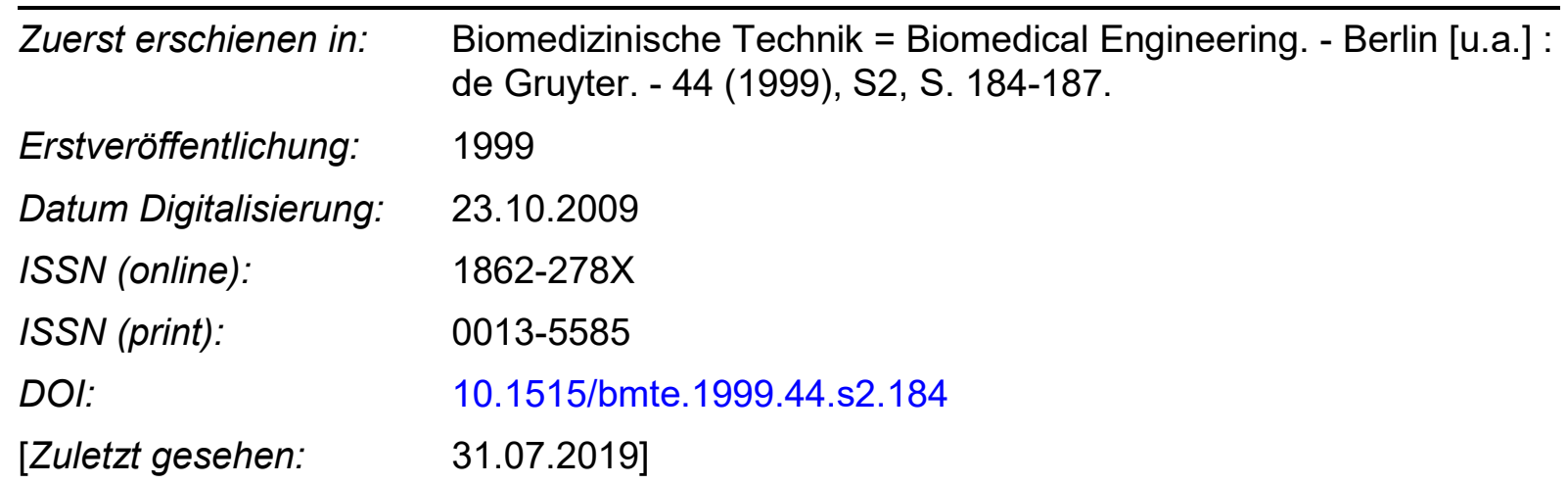

„Im Rahmen der hochschulweiten Open-Access-Strategie für die Zweitveröffentlichung identifiziert durch die Universitätsbibliothek IImenau."

"Within the academic Open Access Strategy identified for deposition by IImenau University Library."

„Dieser Beitrag ist mit Zustimmung des Rechteinhabers aufgrund einer (DFGgeförderten) Allianz- bzw. Nationallizenz frei zugänglich."

"This publication is with permission of the rights owner freely accessible due to an Alliance licence and a national licence (funded by the DFG, German Research Foundation) respectively."

\section{DFG}

Nationallizenzen 


\title{
Physical Phantoms for Biomagnetic and Bioelectric Measurements
}

\author{
Tenner U. ${ }^{1}$, Erné S.N. ', Haueisen J. ${ }^{2}$, Nowak H. ${ }^{2}$, Brauer H. $^{3}$ \\ 'University of Ulm, Ulm, Germany \\ ${ }^{2}$ Friedrich-Schiller-University, Jena, Germany \\ ${ }^{3}$ Technical University of Ilmenau, Ilmenau, Germany
}

\section{Introduction}

The interpretation of the measured, very weak magnetic fields generated by the electrically active organs requires special numerical techniques for localization and reconstruction of the sources. The state-of-the-art of the development of such methods is defined by inverse solution techniques which can reconstruct equivalent dipolar sources within more or less realistically shaped volume conductors. Due to the nonuniqueness of the inverse problem, source reconstruction is only possible under restricted conditions regarding volume conductors and sources. The use of physical phantoms might be helpful to validate the known inverse methods, to improve them or to develop a new one. In addition it is useful to perform magnetic and electrical measurements with physical phantoms under well-defined conditions to validate the models and to calibrate the measuring systems.

One advantage of physical phantom measurements over pure simulation studies, is that the former take into account real world influences such as environmental noise and 3-D positioning errors, thus providing an error estimation of the entire source reconstruction procedure. Current topics of research in bioelectromagnetism are algorithms for reconstruction of extended current sources by solving the inverse problem. The phantoms enables us to perform studies for testing different measuring systems and validating the localization algorithms.

In this paper we present an overview of the four different phantom models, conductivity compartments and five source modules developed in our group within the last three years. The phantoms were filled during measurements with $\mathrm{NaCl}$ solution. A special polymer membrane was used to model different conductive compartments in the thorax. The simplest source model is the current dipole which is a good approximation for focal sources. We developed five special source modules, that can be interchangeably fixed on a ground plate at a defined position at the interior back of the thorax phantom or in the head model. Four of the source modules consist of a set of multiple dipoles. The other module consists of an extended current source (butterfly source model) with variable position and orientation.

Magnetic Resonance Images (MRI) data of the physical phantom models were used to obtain geometric description of the volume conductor for the Boundary Element Method (BEM).

\section{Tank model}

We developed a tank model $(340 \mathrm{~mm}, 310 \mathrm{~mm}$, $190 \mathrm{~mm}$ ) with octagonal cross section for basic studies. It consists of glass and has a plastic ground plate with holes for fixing several configurations of sources, inhomogencities and electrodes for measuring the electrical potentials in space (Fig. 1).

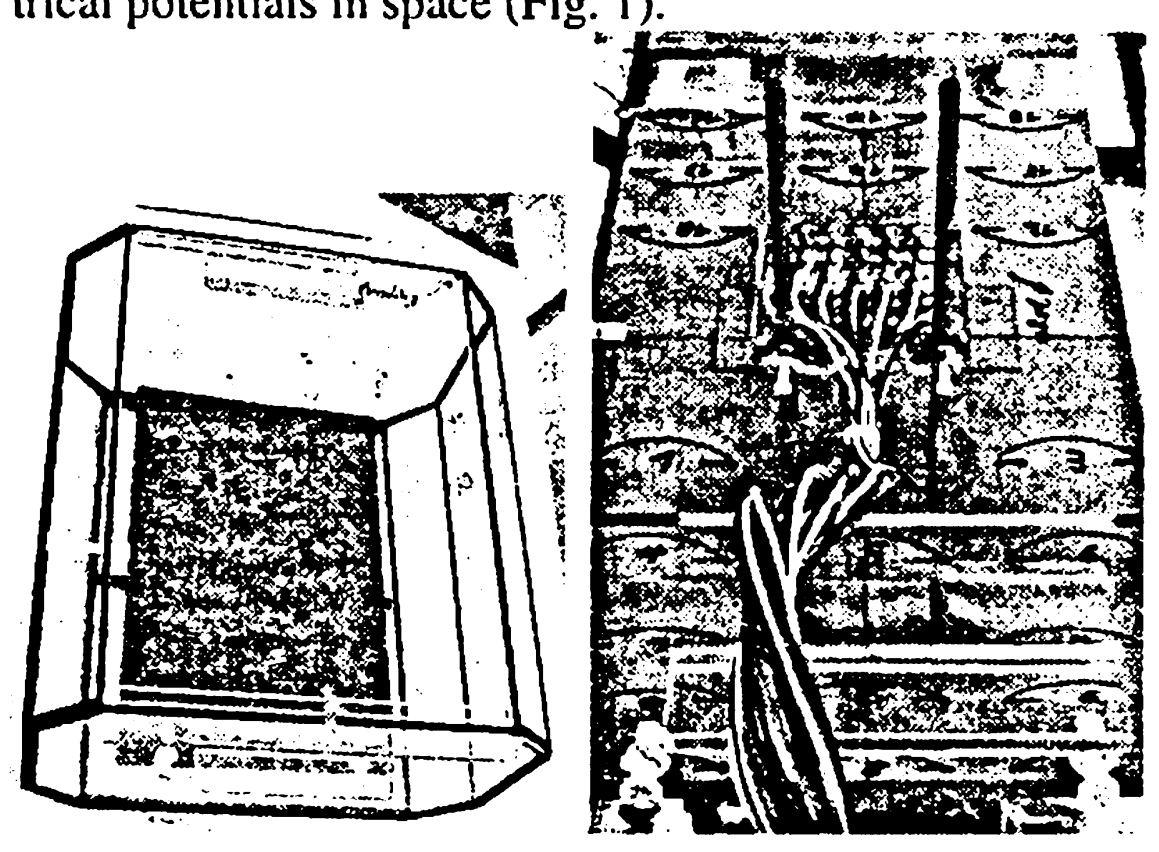

Figure 1: The tank model for basic biomagnetic and bioclectric measurements

We used the tank model for testing of our self designed extended sources and inhomogeneities. An extended current source model was realized by contacting the ends of twisted cooper wires with two thin rectangular plates of platin which were electrically isolated by a plastic cover. Another extended current source model was realized by creating a dipole array with 12 dipoles (Fig. 2).
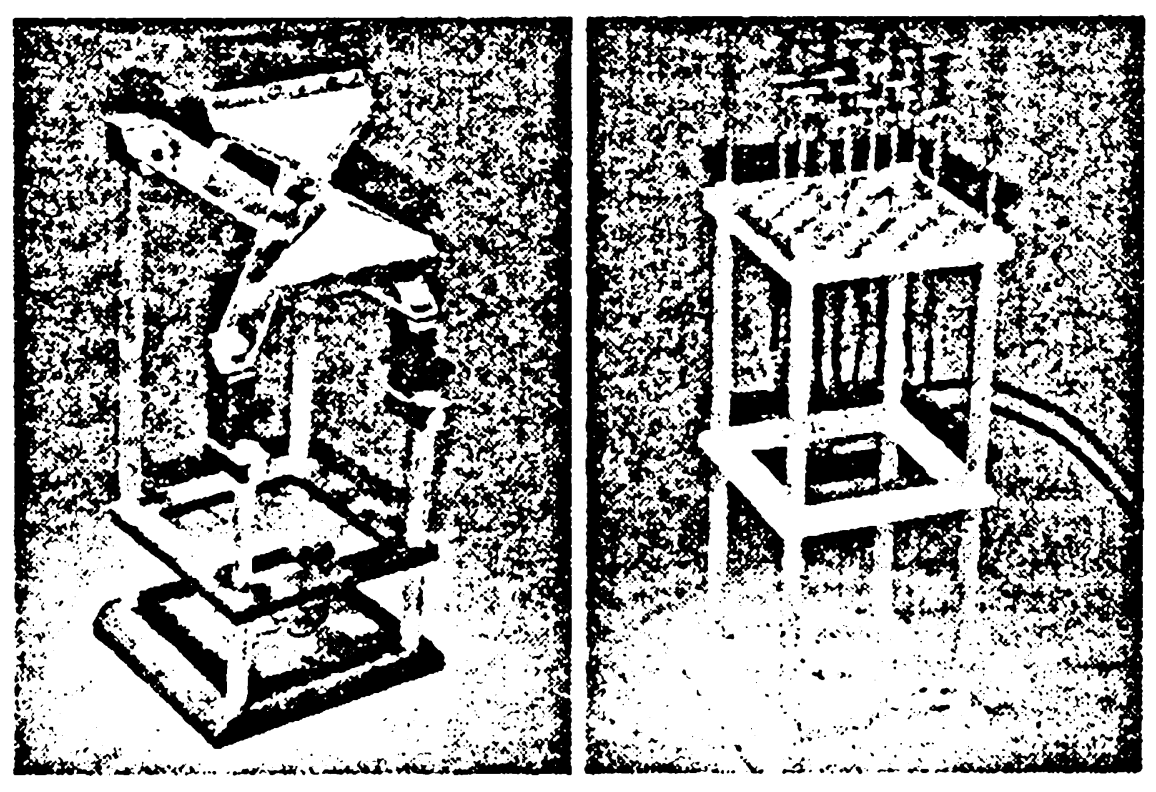

Figure 2: Modules with extended sources, butterfly source (left) and dipole array' (right) 


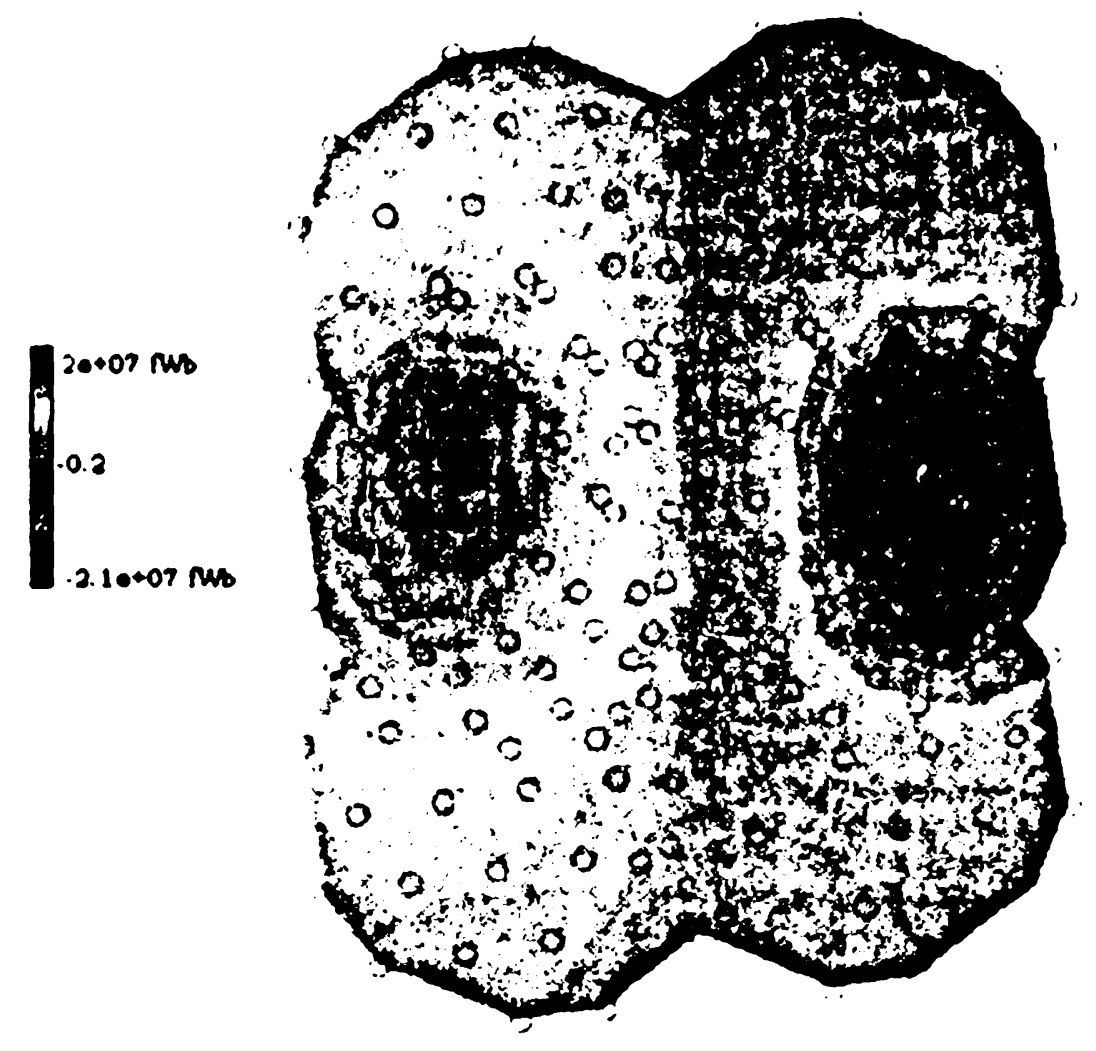

Figure 3: Magnetic field pattern generated by the extended source in the tank model measured with the 31-channel Philips SQUID system at the Biomagnetic Center Jena superimposing the 6 sensor positions and the butterfly source model

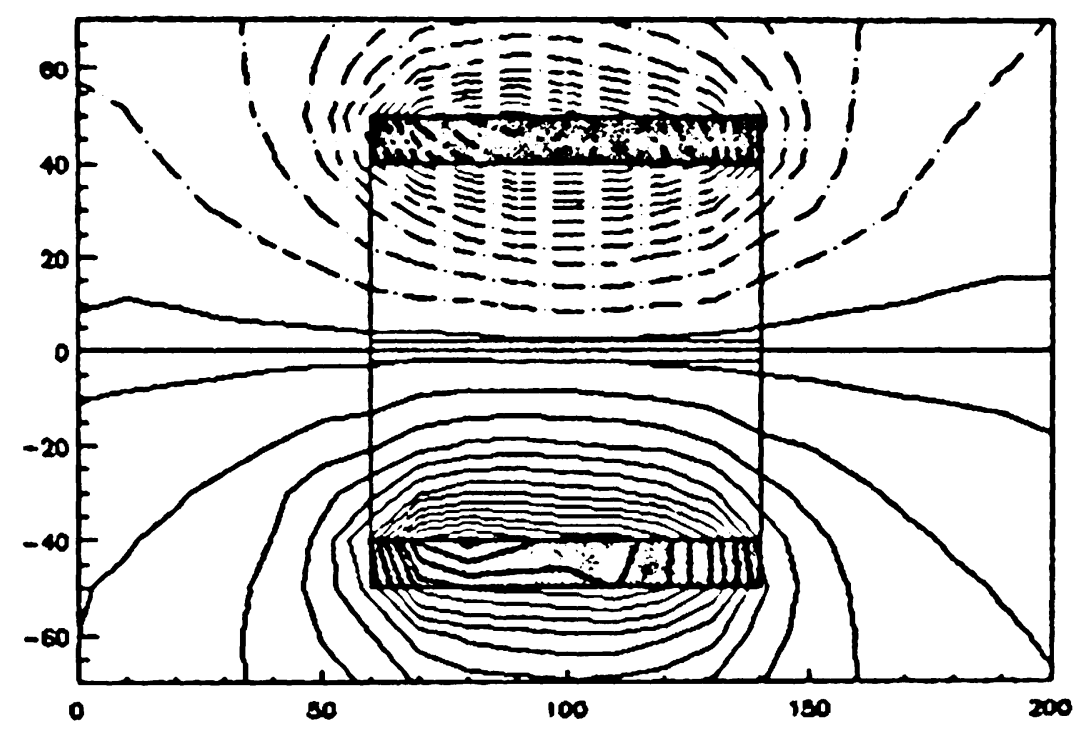

Figure 4: Electrical potential field measured $5 \mathrm{~mm}$ above the source plane

As an example the measurements of the butterfly source in the tank model are shown in Fig. 3 and Fig. 4. Measurements of the magnetic field (about $9 \mathrm{~cm}$ above the current source, Fig. 3) and of the electrical potential field in planes at different heights above the source (Fig. 4 shows the potential field in a distance of $5 \mathrm{~mm}$ to the source) confirm the expecting that the current is almost homogeneously distributed over the platin plates, i.e. this is really an extended source model [1]. Further, we used the butterfly source model in the realistic thorax phantom and in the head model for testing and validating the distributed source reconstruction algorithms [2].

\section{Thorax phantom}

The second model is a realistically shaped thorax phantom including 138 surface electrodes $(\mathrm{Ag} / \mathrm{AgCl})$ to perform magnetic field mapping (MFM) and body surface potential mapping (BSPM) simultaneously as well as to separate magnetic and electrical studies (Fig. 5).
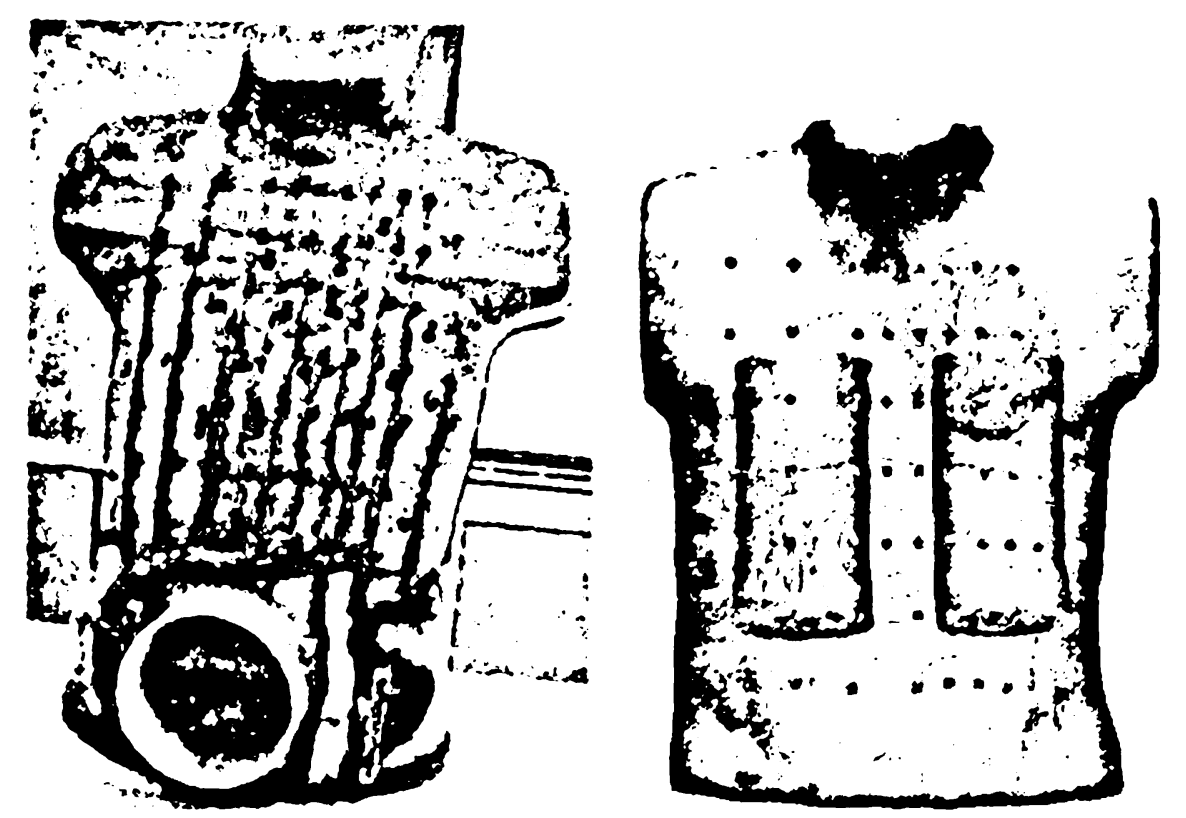

Figure 5: The physical thorax phantom and the inhomogeneous BEM model

The phantom was modeled with epoxy resin by reproducing the body surface of an young adult man. The relatively large filling neck allows the use of different biomagnetic sources and compartments having different conductivity's. A fastening installed on the back of the phantom enables easy and accurate installation and exchange of modules carrying different sources and inhomogeneities, respectively. The cables for the dipole sources can easily be inserted through the opening in the phantom. The firm installation of 138 electrodes $(\mathrm{Ag} / \mathrm{AgCl})$ enables a simultaneous recording of the electric field on the body surface for both the ECG (electrocardiogram) and the BSPM (Body Surface Potential Mapping). In order to obtain a representative result, we followed the NEMY-standard (Non-invasive Evaluation of the MYocardium) [3] with the installation of 120 electrodes.

The artificial lungs were modeled as two cylinders (Fig. 6). The cylinders consist of a plastic rack, and four circular and two rectangular ionic exchangemembranes. The membranes were fixed on the plastic rack to separate the compartments having different conductivity's. Partial compartments of different conductivity's were established by filling the artificial lung with varying $\mathrm{NaCl}$ concentrations [4]. In order to assess the influence of the membrane itself, we compared two measurement series, homogeneous torso without lung membranes and homogeneous torso with lung membranes, filled with the same $\mathrm{NaCl}$ concentration. Between these series we found mean localization differences for the longitudinal oriented dipoles of $0.025 \mathrm{~mm}$ and for the transversal oriented dipoles of $0.348 \mathrm{~mm}$, respectively. This demonstrates that the membrane allows free ionic current flow while keeping the conductivity differences in the compartments. 


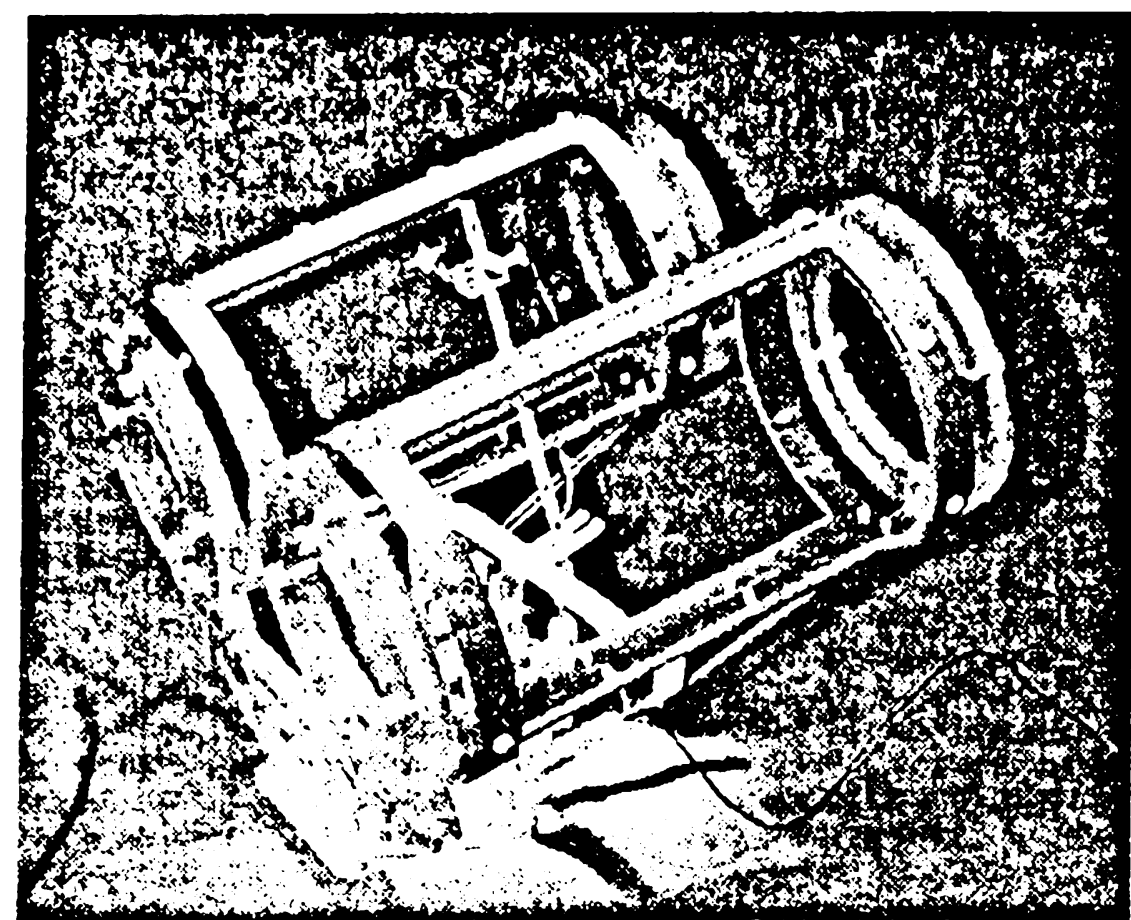

Figure 6: The plastic rack for the artificial lungs

Two dipole orientations were selected at seven different positions (depth): the first orientation longitudinal (craniocaudal) and the second orientation transversal (from right to left). Our main finding was that the accuracy of an estimated source location suffers from the inadequate modeling of the volume conductor, when the artificial lungs were not taken into account for modeling. In those cases both the calculated source location and calculated source strength were misestimated. Mislocalizations of up to $16 \mathrm{~mm}$ and wrong dipole strengths of up to $52 \%$ were obtained. The calculations with the one compartment BEM model of combined MCG/ECG measurements for longitudinal oriented dipoles showed a larger localization error in depth than for transversal oriented dipoles, as the calculated longitudinal oriented dipole positions were ton deep. The main error of the calculated moments is in the direction from the orientation of the dipole. The longitudinal oriented dipoles were too strong, and the transversal oriented dipoles were calculated too weak. We found that the dipole localization error and the calculated dipole strength error did not significantly depend on the dipole depth for longitudinal orientation, but for transversal orientation. The comparison of the maximum and minimum values of the magnetic field with and without artificial lungs for the transversal oriented dipoles shows an obvious influence from the artificial lungs. However, for the longitudinal oriented dipoles no such influence was observed. Also, for the electric potentials no such influence was observed. Additionally we checked the correlation coefficients between magnetic fields and electric potentials with and without artificial lungs. No influence of the artificial lungs was found for the correlation coefficient.

But the comparison of the maximum and minimum values of the magnetic fields with and without artificial lungs from the transversal oriented dipoles shows an obvious influence from the artificial lungs. For the magnetic fields from the longitudinal oriented dipoles and for all electric data no such trend was observed.

For a longitudinal oriented dipole a serious mislocalization in depth was found when omitting the artificial lungs in the BEM model. The dipole was localized on average $11.6 \mathrm{~mm}$ deeper than the actual dipole position. For both the transversal and the longitudinal oriented dipoles, the dipole strength deviates approximately $34 \%$ when omitting the artificial lungs [4].

\section{Spherical model}

The third phantom is a spherical model with a diameter of $67 \mathrm{~mm}$ to characterize the minimum distance resolution between two dipole sources and validate the localization algorithms used in basic research of neuronal processes in animals. The volume conductor consists of two Perspex hemispheres. A special adjustment unit enables us to arrange two or more dipolar sources within $0.1 \mathrm{~mm}$ scale.
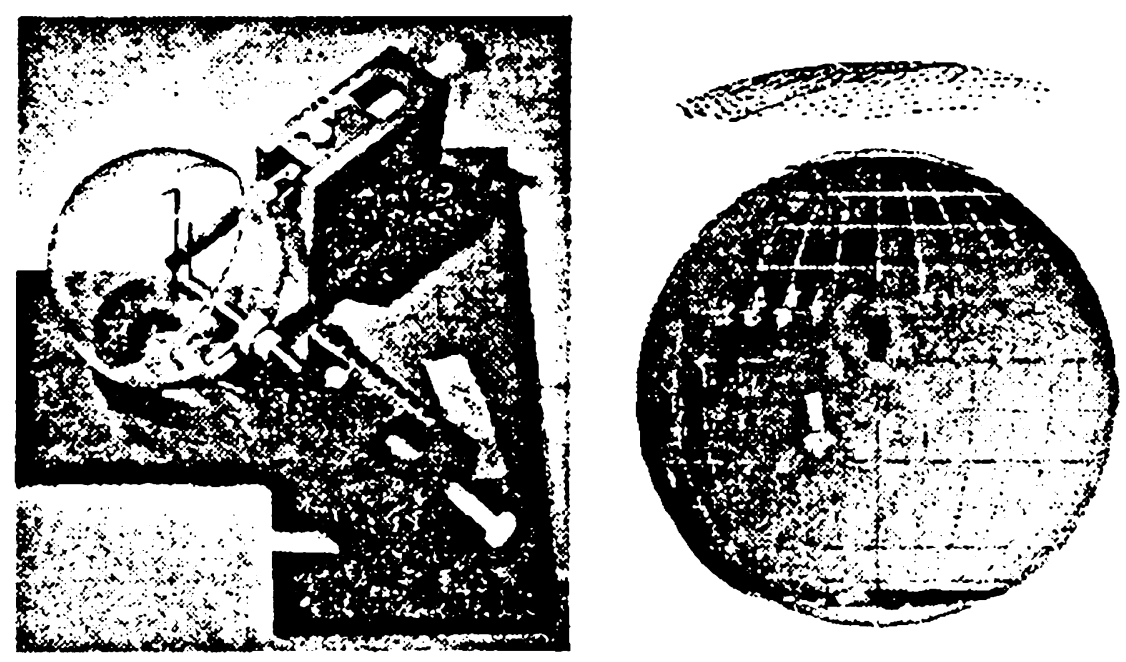

Figure 7: The spherical model with the special adjustment unit (left) and the BEM model (right)

For the verification of the single dipole localization algorithms we used the 31 channel Philips SQUID system. After the entire biomagnetic localization procedure (positioning the model, MCG measuring, creating BEM model from MRI) and by using the Simplex and Levenberg-Marquardt algorithm in the inverse calculations the localization error was less then $1 \mathrm{~mm}$.

For the investigation of the spatiotemporal organization of neuronal processes in animals with the Magnetoencephalographic (MEG), a high temporal resolution (ms) as well as a reasonable spatial resolution of about $1 \mathrm{~mm}$ is necessary. We used the spherical model in order to determine the error for the localization of a single dipole and the resolution of multiple dipoles [5]. The investigation of a 16-channel-Micro-SQUIDMEG-system with a current dipole orientated tangential to the phantom surface produced the following spatial errors ( $x, y$ - horizontal plane, $z$ - depth): systematic localization error $e_{x}=1.16$ to $1.67 \mathrm{~mm}$, $e_{y}=-1.01$ to $-1.28 \mathrm{~mm}, e_{z}=-5.2210-7.64 \mathrm{~mm}$, standard deviation of the single measurements perpendicular to the dipole-axis $\mathrm{s}_{\text {perp }}=0.05$ to $0.22 \mathrm{~mm}$, along to this axis $s_{\text {along }}=0.20$ to $1.73 \mathrm{~mm}$, in the depth $\mathrm{s}_{\mathrm{z}}=0.17$ to $3.17 \mathrm{~mm}$. The "goodness of fit" was better $95 \%$. The separation of two dipoles was still possible for parallel dipoles with a distance of $d_{\text {parallel }}=0.03 \mathrm{~mm}$ and for those perpendicular orientated to each other with a distance of $d_{p e r p}=0.10 \mathrm{~mm}$. The source local- 
ization from the measured magnetic fields has been done by an algorithm of spatial filtering.

\section{Head model}

The fourth phantom is a head model consisting of a plastic cylinder with a rounded top for testing helmet systems (200 mm high, $115 \mathrm{~mm}$ diameter). The ground plate is designed in a way that the source modules of the thorax phantom can be used also in the head phantom.

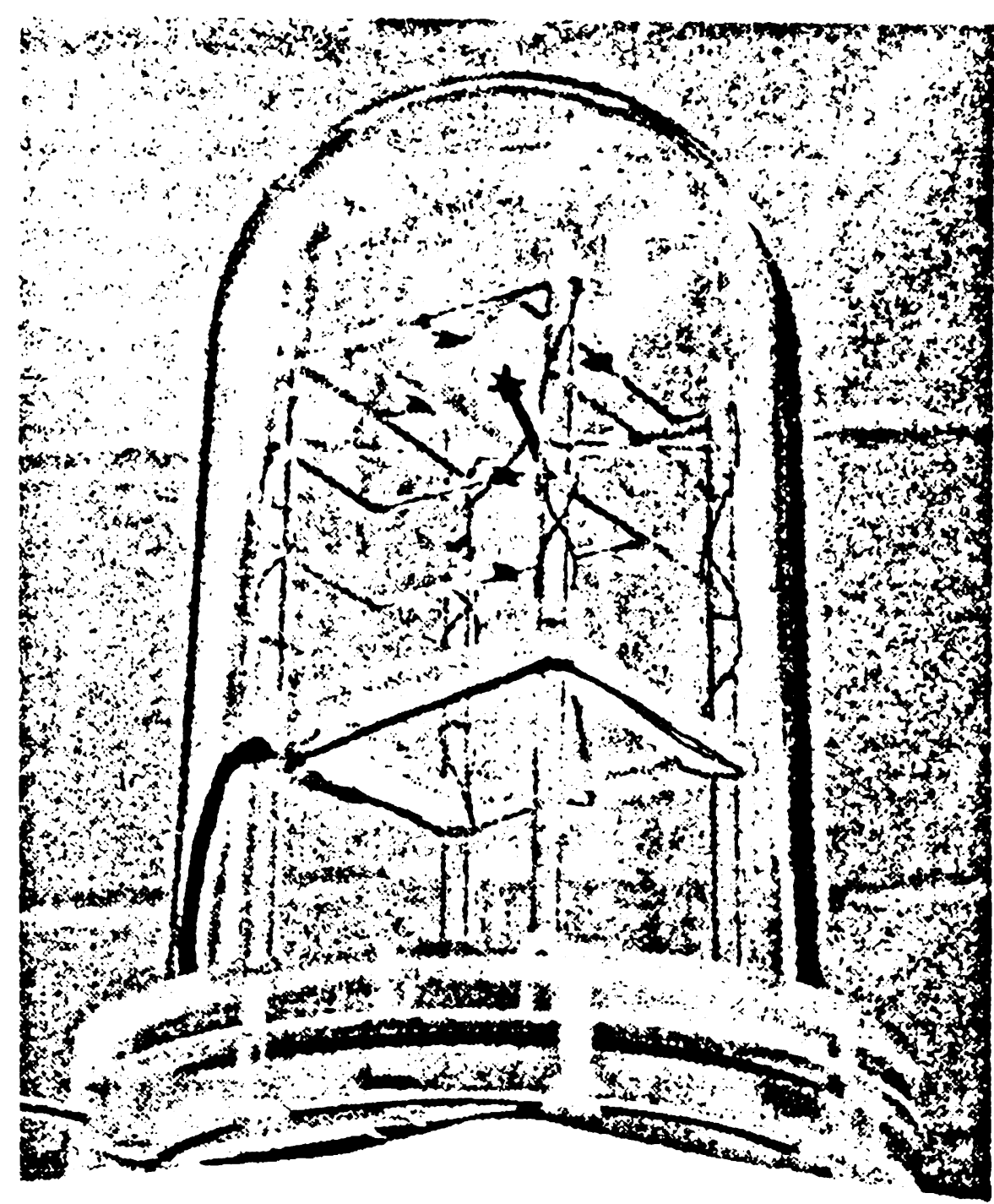

Figure 8: The head model with a 8 dipole source module

This very recent development is now ready to be used in different helmet systems. The production of some copies of this model is planed.

\section{Discussion}

We realized a new physical model of an extended current source which can be used for both biomagnetic and bioelectric studies. Measurements of the magnetic field and the electrical potential field generated by this current source have clearly shown that this model can be used as an extended source model and hence, can be utilized for validating computer simulation methods.

Based on the results with the sphere model we conclude the MEG-system is able to achieve a resolution to investigate neuronal microstructures. The detected spatial errors could be related to positioning of the sen- sors in the cryostat vessel as well as external low frequency noise.

The ionic exchange-membrane does not disturb the free ionic current flow within the volume conductor. Thus, we conclude that the ionic exchange-membrane is well-suited for the construction of conductivity compartments in physical phantoms.

There are certain limitations in the application of our results to real patient data. First, our inhomogeneity modeling is restricted to cylinders, whereas the lungs are of complex shape. Second, in reality, dipoles usually are not longitudinal or transversal orientated. Thus, we believe that omission of the lungs in the forward model will result in a mixture of the different effects on the errors observed in this study. In other words, one would observe a certain instability of the inverse algorithm, where single localization results are not predictable. Nevertheless, this study gives an estimate of the order of the error in the source localization procedure.

The described physical phantoms has proved to be suitable for estimating the accuracy of biomagnetic measuring systems.

\section{References}

[1] Brauer, H., Tenner, U., Wiechmann, H., Ziolkowski, M., Haueisen, J., Nowak, H. and Leder, U., Verification of biomagnetic source reconstruction techniques by using a physical phantom, International Superconductive Electronics Conference, Second Circular (ISEC'97, June 25-28) Berlin, Germany, 1997, $338-340$

[2] Brauer, H., Kosch, O., Ziolkowski, M., Tenner, U. and Haueisen, J., Rekonstruktion verteilter Stromquellen in einem Thoraxphantom mit Hilfe biomagnetischer Feldmessungen, 43. Int. Wiss. Kolloquium, TU Ilmenau, 1998, Proc., 3: 89-94

[3] Hoekema, R., Huiskamp, G. J. M., Oostendorp, T. F., Uijen, G. J. H., and van Oosterom, A., Lead system transformation for pooling of body surfacemap data a surface Laplacian approach, Journal of Electrocardiology, 1995, 28: 344 - 345

[4] Tenner, U., Haueisen, J., Nowak, H., Leder, U. and Brauer, H., Source localization in an inhomogeneous physical thorax phantom, Physics in Medicine and $\mathrm{Bi}$ ology, 1999, 44:

[5] Hagner, T., Eiselt, M., Giessler, F., Hansen, E., Haueisen, J. and Nowak, H., Hochauflösende Magnetoenzephalographie - Untersuchungen mit einem kleinvolumigen Phantom, 1999, 44: 38-45 